\title{
PROSPECÇÃO DE PRODUTOS NATURAIS OBTIDOS DE PRIPRIOCA COMO AGENTES DE CONTROLE BIOLÓGICO DE FUNGOS FITOPATOGÊNICOS DE IMPORTÂNCIA AGRÍCOLA
}

\author{
Aline Aparecida München Kasperi; Sara Freitas de Sousa²; Breno Sena San Martin ${ }^{3}$; José Jeosafá Vieira \\ de Sousa Júnior; ${ }^{4}$ Adilson Sartoratto5; Silvia Katrine Escher ${ }^{6}$; Lauro Euclides Soares Barata ${ }^{7}$. \\ 1 Universidade Federal do Oeste do Pará, Santarém, Pará, Brasil, aliny_@msn.com \\ 2 Universidade Federal do Oeste do Pará, Santarém, Pará, Brasil, sara_freitas_stm@hotmail.com \\ 3 Universidade Federal do Oeste do Pará, Santarém, Pará, Brasil, brenosanmartin95@gmail.com \\ ${ }^{4}$ Universidade Estadual de Campinas, Campinas, São Paulo, Brasil, adilson@cpqba.unicamp.br \\ ${ }^{5}$ Universidade Federal do Oeste do Pará, Santarém, Pará, Brasil, josejeosatajrstm@hotmail.com \\ ${ }^{6}$ Universidade Federal do Oeste do Pará, Santarém, Pará, Brasil, katrineescher@hotmail.com \\ 7 Universidade Federal do Oeste do Pará, Santarém, Pará, Brasil, lauroesbarata@gmail.com
}

RESUMO: Doenças causadas por microrganismos são responsáveis por limitações graves na produção agrícola, e os principais produtos utilizados no controle dos mesmos são compostos químicos sintéticos que possuem aspectos negativos quanto ao seu uso extensivo. Com isso, novos métodos alternativos de controle, como o uso de produtos naturais extraídos de plantas, têm sido investigados devido ao menor impacto ao ambiente. Portanto, neste estudo, objetivou-se caracterizar os metabólitos secundários presentes no óleo essencial e no extrato hexânico do resíduo sólido de priprioca (Cyperus articulatus L.) e a sua capacidade de promover o controle biológico dos fungos Aspergillus niger e Aspergillus parasiticus. Foram coletados tubérculos, rizomas e raízes de priprioca (período chuvoso e seco). O óleo essencial foi extraído do material vegetal por hidrodestilação (6h) em Clevenger. O resíduo sólido foi extraído em Soxhlet (hexano, 8h). A análise química do óleo foi por cromatografia gasosa (CG-EM) e do extrato hexânico por cromatografia de camada delgada (CCD). O ensaio antimicrobiano foi realizado pelo método de difusão em disco. Os compostos majoritários do óleo essencial de priprioca nos dois períodos foram $\alpha$ pineno, trans-pinocarveol, mirtenol, verbenona, mustacona, aristolona e 7isopropenil-1,4a-dimetil-4,4a,5,6,7,8-hexahidro-3H-naftalen-2-ona. No extrato hexânico verificou-se a presença de terpenos e ácidos graxos. O extrato hexânico do resíduo não foi eficiente contra os fitopatógenos. Houve maior inibição para A. niger, quando submetido ao óleo de priprioca extraído no período seco $(18,6 \mathrm{~mm})$. Estes dados indicam o potencial antifúngico da espécie frente a esses fungos sugerindo que a mesma pode ser utilizada para o controle biológico do gênero Aspergillus.

PALAVRA-CHAVE: Controle alternativo, Fitopatógenos, Fungos. 


\title{
PROSPECTION OF NATURAL PRODUCTS OBTAINED FROM PRIPRIOCA AS A BIOLOGICAL CONTROL AGENT OF PHYTOPATHOGENIC FUNGI OF AGRICULTURAL IMPORTANCE
}

\begin{abstract}
Diseases caused by microorganisms are responsible for strong limitations in the production of agricultural, and the main products used in the control them are synthetic chemical compounds that hold negative aspects regarding their extensive use. Thus, new alternative control methods, such as the use of natural products extracted from plants, have been investigated due to the lower impact on the environment. Therefore, in this study, we characterizing the secondary metabolites present in the essential oil, hexanic extract of the solid residue of priprioca (Cyperus articulatus L.) and their ability to promote the biological control of fungi Aspergillus niger and Aspergillus parasiticus. Tubers, rhizomes and roots of priprioca (rainy and dry period) were collected. The essential oil was extracted from the plant material by hydrodistillation (6h) in Clevenger. The solid residue was extracted into Soxhlet (hexane, 8h). The chemical analysis of the oil was by gas chromatography (GC-MS) and the hexane extract by thin layer chromatography (CCD). The antimicrobial test was performed by the disc diffusion method. The major compounds of the essential oil of priprioca in the two periods were $\alpha$-pinene, trans-pinocarveol, mirtenol and verbenone, mustacona, aristolone and 7-isopropenyl-1,4a-dimethyl-4,4a, 5,6,7,8hexahydro-3H-naphthalen-2-one. In the hexane extract the presence of terpenes and fatty acids was verified. The hexane extract from the residue was not efficient against phytopathogens. There was greater inhibition for A. niger, when submitted to the oil of priprioca extracted in the dry period $(18,6 \mathrm{~mm})$. These data indicate the antifungal potential of the species against these fungi suggesting that it can be used for the biological control of the genus Aspergillus.
\end{abstract}

KEYWORDS: Alternative control, Fungi, Phytopathogens.

\section{PROSPECCIÓN DE PRODUCTOS NATURALES OBTENIDOS DE PRIPRIOCA COMO AGENTES DE CONTROL BIOLÓGICO DE HONGOS FITOPATÓGENOS DE IMPORTANCIA AGRÍCOLA}

RESUMEN: Las enfermedades causadas por microorganismos son responsables de limitaciones graves en la producción agrícolas, y los principales productos utilizados en el control de estos son compuestos químicos sintéticos que tienen aspectos negativos en cuanto a su uso extensivo. Con ello, nuevos métodos alternativos de control, como el uso de productos naturales extraídos de plantas han sido investigados debido al menor impacto al ambiente. Por lo tanto, en este estudio, se objetivó caracterizar los metabolitos secundarios presentes en el aceite esencial y en 
el extracto hexánico de priprioca (Cyperus articulatus L.) y su capacidad de promover el control biológico de los hongos Aspergillus niger y Aspergillus parasiticus. Se recogieron tubérculos, rizomas y raíces de priprioca (período lluvioso y seco). El aceite esencial fue extraído del material vegetal por hidrodestilación (6h) en Clevenger. El residuo sólido fue extraído en Soxhlet (hexano, 8h). El análisis químico del aceite fue por cromatografía gaseosa (CG-EM) y el extracto hexánico por cromatografía de capa delgada (CCD). El ensayo antimicrobiano fue realizado por el método de difusión en disco. Los compuestos mayoritarios del aceite esencial de priprioca en los dos períodos fueron $\alpha$-pineno, trans-pinocarveol, mirtenol y verbenona, mustacona, aristolona y 7-isopropenil-1,4a-dimetil-4,4a, 5,6,7,8- hexahidro-3H-naftalen-2-ona. En el extracto hexánico se verificó la presencia de terpenos y ácidos grasos. El extracto hexánico del residuo no fue eficaz contra los fitopatógenos. Se produjo una mayor inhibición para $A$. niger, cuando se sometió al aceite de priprioca extraído en el período seco $(18,6 \mathrm{~mm})$. Estos datos indican el potencial antifúngico de la especie frente a estos hongos sugiriendo que la misma puede ser utilizada para el control biológico del género Aspergillus.

PALABRA CLAVE: Control alternativo, Fitopatógenos, Hongos.

Os fungos fitopatogênicos são responsáveis pela decomposição de substratos celulósicos, ocasionando prejuízos como podridão de sementes, morte de plântulas, tombamento de mudas, estagnação do crescimento, cancros e murcha (BRUTON et al., 2000; MICHEREFF; ANDRADE; MENEZES, 2005; VIDA et al., 2004). A contaminação de produtos agrícolas por fungos produtores de toxinas ocasiona perdas significativas na produtividade, diminuindo valor nutricional dos alimentos e gerando danos à saúde animal e humana (MAZIERO; BERSOT, 2010; SCUSSEL, 2000). Dentre esses microrganismos, fungos do gênero Aspergillus se destacam como produtores de micotoxinas, e por colonizarem frequentemente cereais, grãos e sementes, ocasionam infestações severas a ponto de tornarem os alimentos impróprios para o consumo (APHA, 2001).

Várias estratégias têm sido utilizadas para a obtenção de alimentos saudáveis, desde a produção à 
comercialização, visando suprir as necessidades humanas com abordagens sustentáveis, que possibilitem a interação entre o homem e o ambiente de forma que a ação humana seja componente dos processos ecológicos que acontecem nos agroecossistemas (ALMEIDA, 2012).

Os produtos químicos não renováveis ainda são os mais utilizados para o controle de pragas e doenças de plantas, no entanto possuem aspectos negativos, ou seja, diversos problemas associados ao uso extensivo desses agroquímicos, o que inclui desde a intoxicação de animais e plantas, além da contaminação ambiental. Isso tem levado à procura por métodos alternativos de controle que ocasionem menor impacto ao ambiente, como o uso de produtos naturais extraídos de diferentes plantas (PEREZ; IANNACONE, 2006).

Nesse contexto, a floresta tropical Amazônica, devido a sua grande biodiversidade, apresenta-se como um acervo natural de matérias-primas de grande potencial econômico e ambiental, especialmente quanto a diversidade de espécies vegetais detentoras de metabólitos secundários de atividade biológica com potencial para aplicação biotecnológica, como por exemplo, a priprioca.

Priprioca (Cyperus articulatus) é uma espécie vegetal encontrada na América do Sul, principalmente na região Amazônica, e em vários países da África (Camarão, República da África Central, Gabão, Senegal), sendo utilizada na medicina tradicional para o tratamento de enfermidades, tais como, enxaqueca, dor de estômago, febre, malária, também possuindo propriedades contraceptivas e abortivas (ZOGHBI et al., 2008, NGO BUM et al., 2001).

No estado do Pará, a priprioca, devido suas propriedades aromáticas, é usada na preparação de banhos de cheiro em festas de São João, na aromatização da água de lavagem, na confecção de sachês aromatizados devido a sua fragrância agradável, conhecida como cheiro do Pará. A inserção da priprioca como matériaprima na indústria da perfumaria 
aumentou a demanda da planta, agregando valor ao produto, possibilitando que inúmeras famílias na região Amazônica, no entorno da capital Belém-PA, iniciassem o cultivo desta espécie em escala comercial (ZOGHBI et al., 2008).

Dentre os produtos de interesse obtidos da priprioca, o óleo essencial se destaca por apresentar diversos metabólitos secundários de ação biológica diversificada. Entretanto, no processo de obtenção desse óleo são geradas grandes quantidades de resíduos de biomassa, que podem ser vistos como subprodutos do óleo essencial.

No resíduo sólido foram encontrados metabólitos secundários remanescentes da extração do óleo essencial, como sesquiterpenos, ácidos graxos e esteroides (KASPER, 2016). Assim, a caracterização química e a avaliação da atividade biológica de extratos do resíduo de priprioca têm por finalidade agregar valor aos subprodutos da extração e tornar o processo de obtenção de óleo essencial mais sustentável. Neste contexto, o objetivo do trabalho foi caracterizar os metabólitos secundários presentes no óleo essencial e no extrato hexânico de Cyperus articulatus, visando avaliar a atividade biológica, in vitro, frente aos fungos micotoxigênicos Aspergillus niger e A. parasiticus.

Os tubérculos, rizomas e raízes de priprioca (C. articulatus) foram coletados na Fazenda Experimetal da Ufopa às margens da rodovia CuruáUna, em Santarém, PA. As coletas foram realizadas em períodos sazonais distintos: fevereiro/2017 (período chuvoso) e agosto/2017 (período seco).

O material vegetal (rizomas, tubérculos e raízes) foi lavado em água corrente e seco em estufa a $35^{\circ} \mathrm{C}$ por 72 h, triturado em moinho de facas e o óleo essencial foi extraído em aparelho de Clevenger, por hidrodestilação durante seis horas. O rendimento (\%) dos óleos essenciais foram obtidos em relação a massa seca do material vegetal (DUARTE et al., 2005).

Após a extração do óleo essencial o resíduo sólido foi seco em temperatura 
ambiente e submetido a extração de metabólitos remanescentes presentes na biomassa utilizando o solvente hexano em equipamento de Soxhlet por oito horas. Os extratos em solução foram concentrados em evaporador rotativo e obtidos o rendimento (DUARTE et al., 2005).

Os extratos foram diluídos em diclorometano na concentração 10 mg.mL ${ }^{-1}$ e a análise preliminar das classes de metabólitos foi realizada por cromatografia em camada delgada (CCD) em cromatoplacas de alumínio, sílicagel 60 mesh, marca Merck. Para a revelação de terpenoides e ácidos graxos foi utilizado diclorometano 100\% como fase móvel e vanilina sulfúrica como revelador.

O óleo essencial foi analisado por cromatografia gasosa, utilizando o Cromatógrafo a Gás acoplado a Espectrômetro de Massas Agilent, modelo: HP-6890 acoplado a detector seletivo de massas, nas seguintes condições: coluna capilar HP-5MS (30m x 0,25 mm x 0,25 $\mu \mathrm{m})$, temperatura injetor $=220^{\circ} \mathrm{C}$, temperatura detector $=$ $250^{\circ} \mathrm{C}$, coluna $=60^{\circ} \mathrm{C}, 3^{\circ} \mathrm{C} \cdot \mathrm{min}^{-1}, 240^{\circ} \mathrm{C}$ (20min), gás de arraste $=$ He 1,0 mL.min ${ }^{-}$ 1 .

Foi avaliado a atividade antimicrobiana do óleo essencial e do extrato hexânico do resíduo de priprioca (C. articulatus) sobre os fungos Aspergillus niger e Aspergillus parasiticus, isolados do cultivo de milho e arroz, respectivamente. Os microrganismos fazem parte do acervo do Laboratório de Microbiologia da Universidade Federal do Oeste do Pará - Ufopa. A avaliação da atividade antimicrobiana foi realizada pelo método de difusão em disco (BAUER, 1966), tomando-se como inóculo suspensões fúngicas de $A$. niger e $A$. parasiticus ajustadas em espectrofotômetro na escala de 0,5 MacFarland, com solução fisiológica estéril a 0,9\%, equivalente a $10^{4}$ esporos. $\mathrm{mL}^{-1}$, as quais foram semeadas em placas de Petri contendo meio Sabouraud Dextrose Agar (SDA). Foram então dispostos discos de papel cilíndricos de $6 \mathrm{~mm}$ de diâmetro embebidos com 10uL das amostras do 
óleo essencial e do extrato hexânico na concentração de 100mg. $\mathrm{mL}^{-1}$, e como controle positivo foi utilizado o antifúngico (Azimut ${ }^{\circledR}$ ) e negativo (diclorometano). Os fungos foram incubados à $32^{\circ} \mathrm{C}$ por $96 \mathrm{~h}$, sendo registrados em $72 \mathrm{~h}$ e $96 \mathrm{~h}$ os halos de inibição de crescimento e medidos os diâmetros dos halos de inibição de crescimento em milímetros (mm). 0 delineamento experimental foi inteiramente casualizado, em esquema fatorial (4×2), com três repetições.

Os valores de rendimento de extração foram avaliados por estatística descritiva, em função da média aritmética e desvio padrão. Para os fatores e interação detectados como significativos pelo teste $F(p \leq 0,05)$, foi empregado o teste de Tukey $(p \leq 0,05)$.
O rendimento dos óleos essenciais e dos extratos hexânicos do resíduo sólido de priprioca foi maior para as plantas coletadas no período seco (Tabela 1). Corumbá (2009) relata rendimentos na faixa de 0,45 a 0,80\% para óleo essencial de priprioca coletada em dezembro (período chuvoso) no município Genipaúba (PA) utilizando sistema de arraste a vapor para extração. Kasper (2016) obteve rendimentos de 1,85\% para extratos do resíduo sólido do período chuvoso obtidos em Soxhlet com hexano.

Os compostos majoritários presentes no OE de priprioca tanto no período chuvoso quanto no período seco foram considerados aqueles cuja porcentagem relativa fosse $\geq 3 \%$, sendo descritos na Tabela 2.

Tabela 1. Rendimento médio dos óleos essenciais e dos extratos hexânicos de Cyperus articulatus coletadas nos períodos seco e chuvoso.

\begin{tabular}{lll}
\hline \multirow{2}{*}{ Períodos de Coleta } & \multicolumn{2}{c}{ Rendimento Médio (\%) } \\
\cline { 2 - 3 } & Óleo essencial & Extrato Hexânico \\
\hline Seco & $0,688 \pm 0,0130$ & $2,315 \pm 0,275$ \\
Chuvoso & $0,450 \pm 0,045$ & $1,863 \pm 0,141$ \\
\hline
\end{tabular}


Tabela 2. Compostos majoritários presentes no óleo essencial de Cyperus articulatus.

\begin{tabular}{lcc}
\hline Compostos & Período Seco (\%) & Período chuvoso (\%) \\
\hline$\alpha$-pineno & 5,520 & 4,883 \\
trans-pinocarveol & 5,922 & 4,434 \\
mirtenol & 5,804 & 4,444 \\
verbenona & 3,196 & 3,212 \\
$\alpha$-copaeno & 3,478 & 3,212 \\
$\beta$-selineno & 4,126 & 3,873 \\
óxido de cariofileno & 2,838 & 3,376 \\
aristolona & 4,374 & 5,313 \\
mustacona & 11,042 & 14,046 \\
7-isopropenil-1,4a-dimetil- & & \\
4,4a,5,6,7,8-hexahidro-3H-naftalen- & 7,068 & 9,756 \\
2-ona & & 56,549 \\
\hline Soma dos compostos majoritários & 53,368 & \\
\hline
\end{tabular}

De um modo geral, os constituintes químicos majoritários do óleo essencial de priprioca se mantém constantes nos períodos sazonais (seco e chuvoso). Entretanto, observa-se que a porcentagem relativa dos compostos é variável, não seguindo um padrão de variação.

A mustacona foi a substância majoritária do óleo essencial de C. articulatus neste trabalho, registrandose uma concentração relativa de 11\% no período seco e chegando à 14\% no período chuvoso. Estudos têm relatado a atividade biológica deste sesquiterpeno, como atividade antimalárica e atividade antioncocercose (RUKUNGA et al., 2008; METUGE et al., 2014). De acordo com Zoghbi et al. (2006) o óleo essencial de priprioca é uma mistura complexa de monoterpernos e sesquiterpenos, no qual a mustacona também foi o composto encontrado em maior quantidade (7 a 14\%).

Substâncias como $\alpha$-pineno, transpinocarveol, mirtenol, óxido de cariofileno e $\beta$-selineno também foram caracterizados como compostos majoritários do óleo essencial de priprioca por Zoghbi et al. (2008). No entanto, compostos como epóxido de humuleno, iedol e eudesma-11-dien-5ol não foram encontrados como 
majoritários no óleo essencial de Cyperus articulatus de Santarém-PA. A variação na composição química de óleos essenciais é resultado da influência de diversos fatores bióticos e abióticos, tais como herbivoria, estresse hídrico, solo, temperatura/horário de colheita, a radiação solar, processo de colheita e pós colheita (GLOBBONETO; LOPES, 2007).

A caracterização química dos extratos hexânicos do período seco e chuvoso revelaram terpenos e ácidos graxos com o mesmo padrão de distribuição de substâncias para a fase móvel utilizada. Ngo Bum et al. (1991) relatam a presença de terpernos, flavonoides, catequinas, saponinas e açucares nos tubérculos e rizomas de C. articulatus.

O óleo essencial de C. articulatus apresentou atividade antifúngica sobre A. niger e A. parasiticus (Figura 1).

Figura 1. Ensaio antimicrobiano.

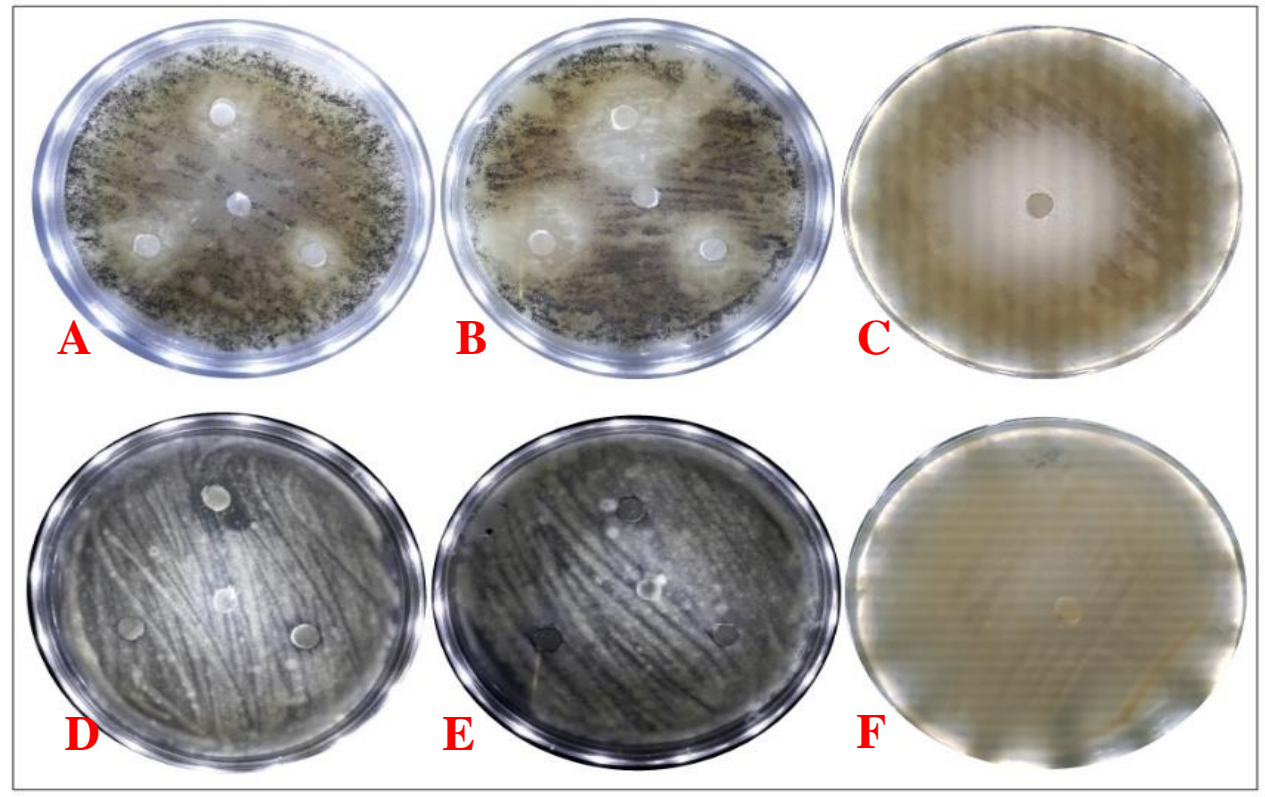

Onde: A) Aspergillus niger submetido ao óleo de priprioca obtido no período chuvoso; B) Aspergillus niger submetido ao óleo de priprioca no período seco; C) Tratamento controle - Aspergillus niger submetido ao fungicida Azimut ${ }^{\circledR}$; D) Aspergillus parasiticus submetido ao óleo essencial de priprioca obtido no período chuvoso; E) Aspergillus parasiticus submetido ao óleo essencial de priprioca obtido no período seco; F) Tratamento controle - Aspergillus parasiticus submetido ao fungicida Azimut ${ }^{\circledR}$. 
No ensaio de antimicrobiano para o fungo A. niger, houve diferença significativa pelo teste de $F$, para $O$ tratamento, tempo e para a interação entre estes, as quais foram desdobradas e analisadas pelo teste de Tukey (Tabela 3).

Ao analisar a interação, evidencia-se que o fungo Aspergillus niger foi mais sensível em 96 h, quando submetido ao tratamento com óleo de priprioca (período seco), com halo de 18,7 mm, o qual diferiu estatisticamente do óleo extraído no período chuvoso com halo de 12,0 mm e dos extratos hexânicos
(Tabela 2). Nabil, Ayman, Ayman (2014) ao avaliarem a eficiência do óleo essencial de priprioca sobre $A$. niger verificaram que o mesmo foi inativo após 24h.

Em comparação com o tratamento controle, os halos de inibição para $A$. niger, observados com o óleo de priprioca obtido no período seco, em 96h, representa aproximadamente 55\% da eficiência do controle químico para este fungo, demonstrando assim, seu potencial de utilização no controle alternativo do patógeno.

Tabela 3. Médias dos halos de inibição proporcionados pelos óleos essenciais e extratos de priprioca, nos diferentes períodos de coleta e tempos de avaliação, para o fungo Aspergillus niger.

\begin{tabular}{lcc}
\hline & \multicolumn{2}{c}{ Halo de Inibição $(\mathrm{mm})$} \\
\cline { 2 - 3 } Produto/Período de Coleta & \multicolumn{2}{c}{ Tempo de Avaliação } \\
\cline { 2 - 3 } & $11,3 \mathrm{aB}$ & $96 \mathrm{~h}$ \\
\hline Óleo essencial/Período seco & $7,8 \mathrm{aB}$ & $18,7 \mathrm{aA}$ \\
Oleo essencial/Período chuvoso & $0,0 \mathrm{bA}$ & $0,0 \mathrm{cA}$ \\
Extrato hexânico/Período seco & $0,0 \mathrm{bA}$ & $0,0 \mathrm{cA}$ \\
Extrato hexânico/Período chuvoso & \multicolumn{1}{c}{ ba } \\
\hline
\end{tabular}

Médias seguidas pelas mesmas letras minúsculas nas colunas e pelas mesmas letras maiúsculas nas linhas não diferem entre si pelo teste Tukey $(p>0,05)$. 
O tratamento com extrato hexânico (período seco e chuvoso) não foi eficiente sobre o gênero Aspergillus spp. avaliados no trabalho, indicado pela colonização fúngica em toda placa nos dois períodos de observação do experimento.

Sousa et al., (2013), ao avaliarem a atividade antimicrobiana do extrato etanólico de priprioca nas concentrações de 5\%; 10\%; 15\%; 20\% e
$50 \%$, sobre isolados do fungo Fusarium spp. provenientes de pimentão, maçaranduba, angelim e angelim da mata, verificaram que a maior concentração foi mais eficiente, com percentual de inibição do crescimento médio de 12,6\%.

O ensaio antimicrobiano com o fungo $A$. parasiticus mostrou resultados significativos pelo teste de F (Tabela 4).

Tabela 4. Médias dos halos de inibição proporcionados pelos óleos essenciais e extratos de priprioca, nos diferentes períodos de coleta e tempos de avaliação, para o fungo Aspergillus parasiticus.

\begin{tabular}{lc}
\hline Tratamento & Halo de inibição $(\mathrm{mm})$ \\
\hline OE (Período seco) & $7,2 \mathrm{~b}$ \\
OE (Período chuvoso) & $10,4 \mathrm{a}$ \\
Extrato hexânico (Período seco) & $0,0 \mathrm{c}$ \\
Extrato hexânico (Período chuvoso) & $0,0 \mathrm{c}$ \\
\hline
\end{tabular}

Médias seguidas pelas mesmas letras minúsculas nas colunas e pelas mesmas letras maiúsculas nas linhas não diferem entre si pelo teste Tukey $(p>0,05)$.

O óleo de priprioca extraído no período chuvoso proporcionou maior controle de A. parasiticus, com halo de inibição de 10,4mm (Tabela 4), representando aproximadamente $80 \%$ da eficiência do produto químico utilizado como controle.
Os óleos essenciais, em sua maioria possuem potencial antifúngico, possivelmente devido à presença de compostos fenólicos e terpenoides (GILLES et al. 2010), sendo que seus constituintes podem atuar como agentes fungistáticos ou fungicidas. Deus et al. 
(2011) comprovaram atividade antifúngica do óleo essencial de copaíba (Copaifera multijuga Hayne) na concentração de $1,6 \mathrm{mg} \mathrm{mL}^{-1}$ contra $A$. flavus, a qual obtiveram halos de inibição do crescimento de 19,5mm. Também foi constatada a atividade inibitória do óleo essencial de orégano (Origanum vulgare) frente ao mesmo fitopatógeno (CLEFF et al., 2010).

Os constituintes químicos majoritários do óleo essencial de priprioca foram constantes nos períodos seco e chuvoso, sendo a porcentagem relativa dos mesmos variável, não seguindo um padrão. Nos extratos hexânicos de ambos os períodos constatou-se terpenoides e ácidos graxos.

O óleo essencial apresentou atividade antifúngica sobre os microrganismos Aspergillus niger e Aspergillus parasiticus avaliados no trabalho, havendo diferença de inibição nos dois períodos estudados (chuvoso e seco). O óleo essencial do período seco foi mais eficiente frente a $A$. niger. $O$ extrato hexânico não foi ativo para os fitopatógenos, o que indica que os compostos responsáveis pela atividade biológica foram extraídos no processo de hidrodestilação do óleo essencial, não estando presentes no extrato hexânico do resíduo sólido.

Nesse contexto, constatou-se o potencial antifúngico in vitro da espécie Cyperus articulatus frente aos fungos avaliados, sugerindo que a mesma pode ser utilizada no controle biológico do gênero Aspergillus, constituindo um recurso alternativo sobre fungos micotoxigênicos de importância agrícola.

\section{AGRADECIMENTOS}

À Universidade Federal do Oeste do Pará por possibilitar o desenvolvimento da pesquisa. Ao CPQBA/UNICAMP pela realização das análises de CG-EM. À Coordenação de Aperfeiçoamento de Pessoal de Nível Superior (CAPES) e ao Fundo de Amparo a Estudos e Pesquisas (FAPESPA) pelo suporte financeiro.

\section{REFERÊNCIAS}

ALMEIDA, J. A. F.; REIS, J. R. M.; LÔPO, C. N. F.; OLIVEIRA, A. S.; FOURNEAU, H. L. Agroecologia. Ihéus: Ceplac/Cenex, 2012, v.1, 44p. 
AMERICAN PUBLIC HEALTH ASSOCIATION - APHA. Compendium of methods for the microbiological examination of foods. 4 ed. Washington, 2001. 676 p.

BAUER, A.; KIRBY, W. M., SHERRIS, J. C.; TURCK, M. Antibiotic susceptibility testing by a standardized single disk method. American Journal of Clinical Pathology, v. 45, n. 4, p. 493, 1966.

BRUTON, B. D.; GÁRCIA-JIMÉNEZ, J.; ARMENGOL, J.; POPHAM, T. W. Assessment of virulence of Acremonium cucurbitacearum and Monosporascus cannonballus on Cucumis melo. Plant Disease, v. 84, p. 907-913, 2000.

CLEFF, M. B.; MEINERZ, A. R.; FARIA, R. O; XAVIER, M. O; SANTIN, R.; NASCENTE, P. S.; RODRIGUES, M.R.; MEIRELES, M.C.A. Atividade inibitória do óleo essencial de orégano em fungos de importância médica e veterinária. Arquivo Brasileiro de Medicina Veterinária e Zootecnia, v. 62, n. 5, p. 1291-1294, 2010.

CORUMBÁ, L. G. Otimização do processo de extração de óleo essencial de priprioca (Cyperus articulatus L.) por arraste com vapor. Belém, 2009, $101 \mathrm{f}$. Dissertação (Mestrado em Engenharia Química), Universidade Federal do Pará, Belém, 2009.

DEUS, R. J. A.; ALVES, C. N.; ARRUDA, M. S. P. Avaliação do efeito antifúngico do óleo resina e do óleo essencial de copaíba (Copaifera multïuga Hayne). Revista Brasileira de Plantas Medicinais, v. 13, n. 1, p. 1-7, 2011.
DUARTE, M. C. T.; FIGUEIRA, G. M.; SARTORATTO, A.; REHDER, V. L. G.; DELARMELINA C. Anti-candida activity of Brazilian medicinal plants. Journal of Ethnopharmacology, v. 97, p. 305-311, 2005.

GILLES, M.; ZHAO, J.; AN, M.; AGBOOLA, S. Chemical composition and antimicrobial properties of essential oils of three Australian Eucalyptus species. Food Chemistry. v. 119, p. 731-737, 2010.

GLOBBO-NETO, L.; LOPES, N. P. Plantas Medicinais: Fatores de Influência no Conteúdo de Metabólitos Secundários. Química Nova, v. 30, n. 2, p. 374-381, 2007.

KASPER, A. A. M. Estudo fitoquímico e avaliação da atividade biológica do resíduo sólido e do hidrolato dos rizomas de priprioca (Cyperus articulatus var. nodosus) cultivada em Santarém-PA, Brasil. Santarém, 2016. 128 f. Dissertação (Mestrado em Ciências Ambientais). Universidade Federal do Oeste do Pará, Santarém, 2016.

MAZIERO, M. T.; BERSOT, L. S. Micotoxinas em alimentos produzidos no Brasil. Revista Brasileira de Produtos Agroindustriais, v. 12, p. 89-99, 2010.

METUGE, J. A.; BABIAKA, S. B.; MBAH, J. A.; NTIE-KANG, F.; AYIMELE, G. A.; CHONGWA, F. Anti-onchocerca Metabolites from Cyperus articulatus: Isolation, In Vitro Activity and In Silico 'DrugLikeness', Natural Products Bioprospecting, v. 04, p. 243-249, 2014. 
MICHEREFF, S. J.; ANDRADE, D. E. G. T.; MENEZES, Maria. Ecologia e manejo de patógenos radiculares em solos tropicais. Universidade Federal e Rural de Pernambuco, 2005.

NABIL A. E.; AZZAZ, A. Y.; EL-KHATEEB, A. A. F. Chemical composition and biological activity of the essential oil of Cyperus articulatus. International Journal of Academic Research. v. 6, p. 265-269, 2014.

NGO BUM, E; RAKATONIRINA, S. V.; EFA, F.; MAHAILET, J. B. Contribution à l'étude de l'activité psychotrope de l' extrait de Cyperus articulatus. Biociences Proceedings, v. 2, p. 286-288, 1991.

NGO BUM, E.; SCHMUTZ, M.; MEYER, C.; RAKATONIRINA, A.; BOPELET, M.; PORTET, C; JEKER, A..; RAKOTONIRINA, S. $V_{i}$; OLPE, H. R.; HERRLING, P. Anticonvulsant properties of the methanolic extract of Cyperus articulatus (Cyperaceae), Journal of Ethnopharmacology, v. 76, p. 145- 150, 2001.

PEREZ, D. D.; IANNACONE, J. O. Efectividad de extractos botánicos de diez plantas sobre la mortalidad y repelencia de larvas de Rhynchophorus palmarum L., insecto plaga del pijuayo Bactrisga sipaes Kunth. en la Amazonia del Perú. Agricultura Técnica, Lima, v. 66, p. 21-30, 2006.

RUKUNGA, G. M.; MUREGI, F. W.; OMAR, S. A.; GATHIRWA, J. W.; MUTHAURA, C. N.; PETER, M. G.; HEYDENREICH, M.; MUNGAI, G. M. Anti-plasmodial activity of the extracts and two sesquiterpenes from Cyperus articulates, Fitoterapia, p. 188-190, 2008.

SCUSSEL, V. M. Atualidades em micotoxinas e armazenagem de grãos. Florianópolis: Insular, 2000. v. 1, 382 p.

SOUSA, B.C.M.; LUSTOSA, D.C.; RODRIGUES, A.S.; BARATA, L.E.S.; CASTRO, K.C.F., AREVALO, M.R. Extrato de priprioca (Cyperus articulatus L,) no controle de isolados de Fusarium spp. In: SIMPÓSIO BRASILEIRO DE ÓLEOS ESSENCIAIS, 7., Santarém, 2013. Anais... Santarém: UFOPA, 2013.

VIDA, J. B.; ZAMBOLIM, L.; TESSMANNI, D. J.; BRANDÃO FILHO, J. U. T.; VERZIGNASSI, J. R.; CAIXETA, M. P. Manejo de doenças de plantas em cultivo protegido. Fitopatologia Brasileira, v. 29, n. 4, p. 355-372, 2004.

ZOGBHI, M. G. B.; ANDRADE, E. H. A.; OLIVEIRA, J.; CARREIRA, L.M. M.; GUILHON, G. M. S. P. Yield and chemical composition of the essential oil of the steams and rhizomes of Cyperus articultatus L. cultivated in the state of Para, Brazil, Journal of essential oil, v. 18, 2006.

ZOGBHI, M. G. B.; GUILHON, G. M. S. P.; ANDRADE, E. H. A.; VILHENA, K. S. S. Química das espécies de Cyperus conhecidas por Priprioca In: POTIGUARA, R. C. V.; ZOGHBI, M. G. B. (Org) Priprioca: um recurso aromático do Pará Belém: MPEG, UEPA, 2008, p. 53-76. 